\title{
Etiology of cervicitis and treatment with minocycline
}

\author{
WiLliam R BOWIE, MD, VAL WILletts, RN, Bernard A BINNS, MD, Robert C BRUNHAM, MD
}

WR BOWIE, V WILLETTS, BA BINNS, RC BRUNHAM. Etiology of cervicitis and treatment with minocycline. Can J Infect Dis 1993;4(2):95-100.

Oвлестіv: To evaluate the etiology of cervicitis using the recommended Canadian definition, and to evaluate the efficacy and tolerability of seven days of minocycline treatment, 100 versus $200 \mathrm{mg}$ at bedtime. Design: Randomized double-blind study with initial microbiological evaluation, and intended follow-up through 12 weeks. Serting: Women attending the major sexually transmitted disease clinic in Vancouver and the major teaching hospital in Winnipeg. Population STUdied: Women with cervicitis (inclusion criteria were an off-white or yellow colour of cervical mucus when viewed on a white-tipped swab, and a mean of 10 or more polymorphonuclear leukocytes per oil immersion [x 1000] field on Gram stain of cervical mucus]. Fourty-four women were enrolled but two were excluded because of contaminated cultures. InTERvENTIONs: Treatment with two identical appearing capsules of $50 \mathrm{mg}$ ( $100 \mathrm{mg}$ dose) or $100 \mathrm{mg}$ ( $200 \mathrm{mg}$ dose) of minocycline taken at bedtime with water for seven days. Mann Results: Of the 42 evaluable women, Chlamydia trachomatis was initially isolated from $19(45 \%)$ and Neisseria gonorrhoeae from four (10\%). The study was prematurely terminated because of an unacceptable and significantly higher frequency of adverse reactions on the higher dose regimen of minocycline - severe reactions in one (4\%) on $100 \mathrm{mg}$ compared with six (30\%) on $200 \mathrm{mg}$ $(\mathrm{P}=0.05)$. Major reactions were dizziness, mood alterations and nausea. Clinical parameters, but not numbers of polymorphonuclear leukocytes, improved significantly irrespective of initial microbiology or the regimen received. Cultures became and stayed negative for $C$ trachomatis in seven of eight on minocycline $100 \mathrm{mg}$ and five of six on minocycline $200 \mathrm{mg}$. Both 'failures' had an intervening negative culture and were re-exposed to untreated sexual partners. Conclusions: Although not a definitive study in terms of proving efficacy of lower dose regimens, the results are consistent with efficacy and demonstrate the significant advantage of the lower dose regimen in terms of adverse reactions.

Key Words: Cervicitis, Chlamydia trachomatis, Neisseria gonorrhoeae, Minocycline

\section{Étiologie de la cervicite et son traitement avec la minocycline}

Овлестг : Évaluer l'étiologie de la cervicite au moyen de la définition canadienne recommandée, ainsi que l'efficacité et la tolérance d'un traitement de minocycline pendant sept jours, à raison de 100 mg comparé à $200 \mathrm{mg}$ administré à l'heure du coucher. MérHodologre : Étude en double insu randomisée avec évaluation microbiologique initiale, et suivi prévu sur 12 semaines. CADRE : Femmes fréquentant la principale clinique de maladies transmises sexuellement de Vancouver et le principal hôpital universitaire de Winnipeg. Population étudiée : Femmes atteintes de cervicite. Conditions Reguises : Couleur blanchâtre ou jaune du mucus cervical examiné sur un écouvillon blanc, et une moyenne de dix (ou plus) leucocytes polynucléaires par champ microscopique à immersion ( $X 1000)$ d'étalement cervical sous coloration de Gram. Quarante-quatre femmes ont été inscrites, mais deux ont été exclues à cause de la contamination de la culture. InTERVEnTIONs : Traitement avec deux capsules d'aspect identique de $50 \mathrm{mg}$ (dose totale 100 $\mathrm{mg}$ ) ou $100 \mathrm{mg}$ (dose totale $200 \mathrm{mg}$ ) de minocycline prises au moment du coucher avec de l'eau, pendant

Division of Infectious Disease, Department of Medicine, University of British Columbia, Vancouver, British Columbia; and the Departments of Obstetrics and Gynecology, Medicine, and Microbiology and Infectious Diseases, University of Manitoba, Winnipeg, Manitoba

Correspondence: Dr William R Bowie, Division of Infectious Diseases, GF Strong Research Laboratory, Room 452,

D Floor, Vancouver General Hospital, 2733 Heather Street, Vancouver, British Columbia V5Z 3J5. Telephone (604) 875-4147

Received for publication February 17, 1992. Accepted May 27, 1992 
7 jours. RÉsultats PRincipaux : Sur les 42 femmes évaluables, 19 (45\%) ont donné au commencement des isolats de Chlamydia trachomatis et 4 (10\%) des isolats de Neisseria gonorrhoeae. L'étude a été abandonnée prématurémant à cause d'une fréquence significative et inacceptable de réactions indésirables aux doses supérieures de minocycline (réactions graves chez une femme $(4 \%)$ avec la posologie de $100 \mathrm{mg}$, et chez six femmes ( $30 \%$ ) avec une posologie de $200 \mathrm{mg}, \mathrm{P}=0,05)$. Les principales réactions étaient les suivantes vertiges, altération de l'humeur et nausées. Les paramètres cliniques (sauf le nombre des leucocytes polynucléaires) se sont améliorés de façon significative indépendamment de la microbiologie initiale ou du traitement reçu. Les cultures sont devenues $C$ trachomatis négatives chez sept femmes sur huit avec 100 mg de minocycline, et chez cinq femmes sur fix avec $200 \mathrm{mg}$ de minocycline. Les deux "échecs" ont fini par avoir une culture négative et ont été réexposés à des partenaires sexuels non traités. ConcLusıon : Bien que l'étude n'ait pas prouvé de façon concluante l'efficacité du traitement à faible dose, ses résultats sont en faveur de son efficacité et démontrent l'avantage important de la posologie plus faible sous le rapport des réactions indêsirables.

$\mathrm{C}$ ERVICITIS IS A FREgUENT AND IMPORTANT INFECTION IN women that is associated with both short and long term complications. The major recognized etiologies of cervical inflammation are Chlamydia trachomatis and Neisseria gonorrhoeae (1). Herpes simplex virus causes an erosive exocervicitis, but that diagnosis usually is readily apparent. Unfortunately, in many women with cervical inflammation and a polymorphonuclear leukocyte response in endocervical secretions, the etiology is not apparent despite extensive studies (2).

Although many individuals infected with $C$ trachomatis and $N$ gonorrhoeae lack symptoms and signs of infection, some do have clinically detectable evidence of infection (3) and guidelines for management of sexually transmitted diseases include suggested criteria for recognition of cervical infection (4-6). However, American and Canadian guidelines differ. The more rigorous definition is that proposed by the Centers for Disease Control in Georgia, which requires evidence of a purulent or mucopurulent endocervical discharge, erythema or edema in an area of ectopy, induced mucosal bleeding (friability) and the presence of 10 or more polymorphonuclear leukocytes per oil immersion $(x 1000)$ field of cervical mucus (4). This is often associated with the presence of $C$ trachomatis, and is infrequently associated with detection of $N$ gonorrhoeae (3). However, less rigorous and perhaps more useful for detection of larger numbers of women with a sexually transmitted cervical infection is the Canadian requirement for only a purulent or mucopurulent endocervical discharge plus increased numbers of polymorphonuclear leukocytes, without other evidence of cervical inflammation $(5,6)$. The expectation is that a lower proportion of these women will have infection with $C$ trachomatis, but more women with gonococcal infection will be recognized. This less rigorous definition may be especially useful in populations at greater risk for sexually transmitted diseases, whereas the more rigorous definition may be needed in other populations to increase the predictive value of positive findings.

Minocycline is very active against $C$ trachomatis in vitro and in vivo (7). Although treatment of uncomplicated $C$ trachomatis genital infection is customarily tetracycline $500 \mathrm{mg}$ orally four times daily for seven days, or doxycycline $100 \mathrm{mg}$ orally twice daily for seven days (4-6), many studies have shown that lower doses are effective $(7,8)$. In men, minocycline $100 \mathrm{mg}$ once daily was as effective as $100 \mathrm{mg}$ twice daily and was much better tolerated (8).

The objectives of this study were to evaluate the etiology of cervicitis (using the less rigorous definition) in two Canadian centres, and to evaluate the efficacy and tolerability of seven days of minocycline treatment, 100 versus $200 \mathrm{mg}$ at bedtime in a double-blind study. The study was prematurely terminated because of an unacceptable frequency of adverse reactions in the higher dose group.

\section{MATERIALS AND METHODS}

Patient population: Women spontaneously presenting to the Vancouver Sexually Transmitted Disease Clinic or the Winnipeg Health Sciences Centre were eligible for enrollment. Inclusion criteria were age over 16 years and clinical criteria for the diagnosis of cervicitis (both an off-white or yellow colour of cervical mucus when viewed on a white-tipped swab and a mean of 10 or more polymorphonuclear leukocytes per oil immersion [x1000] field on Gram stain of cervical mucus which contained less than 100 vaginal squamous epithelial cells when collected from a nonmenstruating woman). Women were excluded if they had received antimicrobials in the preceding month, were pregnant. gave birth or had an abortion in the past six weeks, were menstruating, were allergic to tetracyclines, were using an intrauterine device, had clinical evidence of acute pelvic inflammatory disease, or were known to be infected with $N$ gonorrhoeae, Trichomonas vaginalis, or herpes simplex virus prior to enrollment.

Clinical evaluation: Women were questioned about symptoms and sexual history, and underwent a routine genital and speculum examination. Special attention was paid to the presence of endocervical secretions, edema and erythema in the presence of an area of ectopy, and induced mucosal bleeding with the first cervical swab. The area of ectopy was estimated as a percentage of the area of the portio of the cervix, and 
erythema and edema in an area of ectopy were graded from 0 (normal or none) to 3 (severe). A bimanual examination was performed to specifically assess cervical motion, and uterine and adnexal tenderness.

Collection of specimens: Prior to inserting the speculum, a urethral swab was obtained for Gram stain and for $N$ gonorrhoeae culture. A second urethral swab (calcium alginate) was obtained for $C$ trachomatis culture. A third urethral swab was obtained for genital mycoplasma culture. Vaginal swabs for vaginal Gram stain were assessed for microbial flora, saline preparation, potassium hydroxide preparation, cultures for $T$ vaginalis and cultures for genital mycoplasmas. A swab placed into Amies' transport media for a routine culture was obtained to detect vaginal aerobes and yeasts. After examining the appearance of the cervix, secretions overlying the cervical os were removed by one or more swabs. An endocervical swab was then obtained for Gram stain to evaluate the number of polymorphonuclear leukocytes and to look for Gram-negative intracellular diplococci. A second endocervical swab was obtained for $N$ gonorrhoeae culture and a third endocervical swab (calcium alginate) was obtained for C trachomatis culture. A fourth endocervical swab was obtained and placed in transport media for culture for herpes simplex virus and cytomegalovirus. A Papanicolaou (Pap) smear was also obtained. Pharyngeal and rectal swabs were then obtained for cultures for $N$ gonorrhoeae and $C$ trachomatis (calcium alginate swabs were used for C trachomatis).

Serum was obtained for syphilis serology, erythrocyte sedimentation rate and measurement of antibody to $C$ trachomatis.

Treatment and follow-up: After obtaining specimens, women were given medication which was either two identical-appearing capsules of $50 \mathrm{mg}$ (100 $\mathrm{mg}$ dose) or $100 \mathrm{mg}$ ( $200 \mathrm{mg}$ dose) of minocycline, to take at bedtime with one to two glasses of water for seven days. The allocation of medication was computer randomized and double-blinded.

Women were requested to return for follow-up at approximately eight days, and four, eight and 12 weeks after initiating therapy. Visits were scheduled to avoid menstrual periods. At each follow-up visit women were questioned about symptoms, use of other medications, sexual activity, treatment status of partners and use of barrier methods if sexually active. In addition, at the first visit women were also questioned about compliance with medications and development of adverse reactions. Women were evaluated as initially at all follow-up visits with the exception that Pap smears were only repeated at four and 12 weeks, and pregnancy tests and syphilis serology were only performed at the initial visit.

Microbiological evaluation: Microbiological evaluations, summarized below, were performed using standard techniques (9).
Saline and potassium hydroxide preparations were interpreted within 15 mins. Gram stains were evaluated for number of polymorphonuclear leukocytes and bacteria. To enumerate the number of polymorphonuclear leukocytes in endocervical secretions only areas of mucus without epithelial cells were evaluated.

Specimens for $\mathrm{N}$ gonorrhoeae were cultured and isolates were identified using standard techniques. Specimens for $C$ trachomatis were placed into 2-sucrose phosphate transport medium, frozen at $-70^{\circ} \mathrm{C}$ within $2 \mathrm{~h}$, and thawed and cultured in cycloheximide-treated McCoy cells in vials. Inclusions were identified by iodine staining, with passage of positive specimens to demonstrate that identified inclusions could be passed and with passage of negative specimens to increase the sensitivity of the system. Specimens for genital mycoplasmas were streaked on A7 agar and then placed into U9 broth at the bedside, and then transported to the laboratory within 30 mins. Serial dilutions were then made in U9 broth. Ureaplasma urealyticum was identified on the basis of colour change in broth in conjunction with typical colonial morphology on agar. Large colony mycoplasmas were identified on the basis of typical morphology on agar. Specimens for T vaginalis were placed, at the bedside, into TYI-S-33 medium. Specimens were transported to the laboratory within 30 mins and then incubated. Specimens for viral culture were transported to the laboratory within $2 \mathrm{~h}$ and inoculated into Viro cells.

Antibody to $C$ trachomatis was detected using the modified indirect microimmunofluorescent assay described by Wang et al (10).

Pap smear interpretation: Smears were interpreted using standard methodologies by one cytopathologist (11).

Consent and management of partners: All women gave written informed consent. The protocol was approved by the Human Subjects Review Committees at both centres. When possible, the patients' partners were evaluated and treated.

Criteria for response: Clinical response was primarily assessed on the basis of objective findings. Total response was defined as the disappearance of mucopurulent discharge and, if initially present, disappearance of edema and erythema in an area of ectopy, and disappearance of induced mucosal bleeding. Improvement was defined as a lessening of the discharge and edema, erythema and induced mucosal bleeding. Failure was defined as the absence of improvement.

Microbiological response was defined for those organisms detected at the initial visit. Cure was defined as the disappearance of the organism at the first and all subsequent follow-up visits. Recurrence was defined as a negative culture or diagnostic test at the first followup visit but with a subsequent positive culture or test. Failure was defined as the persistence of a positive culture or test at the first follow-up visit. Recurrences or failures were considered to be regimen failures if the 
TABLE 1

Isolation of genital pathogens at initial visit

\begin{tabular}{|c|c|c|c|}
\hline & \multicolumn{3}{|c|}{ Chlamydia trachomatis } \\
\hline & Present* & Absent $^{\dagger}$ & Total \\
\hline Neisseria gonorrhoeae & $1(5 \%)$ & $3(13 \%)$ & $4(10 \%)$ \\
\hline Ureaplasma urealyticum & $18(95 \%)$ & $17(74 \%)$ & $35(83 \%)$ \\
\hline $\begin{array}{l}\text { Large colony } \\
\text { mycoplasmas }\end{array}$ & $9(47 \%)$ & $11(48 \%)$ & $20(48 \%)$ \\
\hline Gardnerella vaginalis & $14(74 \%)$ & $14(61 \%)$ & $28(67 \%)$ \\
\hline Yeasts & $3(16 \%)$ & $3(13 \%)$ & $6(14 \%)$ \\
\hline Herpes simplex virus & $1(5 \%)$ & 0 & $1(2 \%)$ \\
\hline Trichomonas vaginalis & $1(5 \%)$ & 0 & $1(2 \%)$ \\
\hline Cytomegalovirus & 0 & 0 & 0 \\
\hline
\end{tabular}

\section{TABLE 2}

Detection of erythema, edema and/or induced mucosal bleeding (friability) in women according to initial microbiology

\begin{tabular}{lcc}
$\begin{array}{l}\text { Presence of erythema, } \\
\text { edema and/or friability }\end{array}$ & $\begin{array}{c}\text { Chlamydia trachomatis } \\
\text { Present }^{*}\end{array}$ & $\begin{array}{c}\text { Absent } \\
{ }^{\dagger}\end{array}$ \\
\hline None & 2 & 3 \\
One & 3 & 11 \\
Two & 6 & 4 \\
All three & 8 & 5 \\
\hline
\end{tabular}

${ }^{*} n=19 ;{ }^{\dagger} n=23 ; P=0.05$, two-tailed Fisher exact test, no or one manifestation compared with two or all three manifestations

patients took the medication and had not resumed having sexual intercourse, or had only resumed sexual intercourse with a treated partner or when a condom had been consistently used.

\section{RESULTS}

Thirty-seven women in Vancouver and seven in Winnipeg were enrolled, but two were subsequently excluded from microbiological evaluation because of yeast contamination of cultures for $C$ trachomatis. The two women were included in the analysis of adverse reactions; one was the only woman in the study who had reactive syphilis serology.

Three women failed to return for any follow-up evaluations, although all were contacted by telephone and data on adverse reactions were obtained. Failure to return was not attributed to adverse reactions. Two or more follow-up visits were made by 32 patients.

Initial microbiological results: $C$ trachomatis was isolated from 19 women (45\%) and $\mathrm{Ngonorrhoeae}$ from four $(10 \%)$. Table 1 shows the microbiological results according to whether $C$ trachomatis was isolated. The distribution of other agents was similar whether $C$ trachomatis was detected.

Clinical observations: By definition, all women had cervicitis. Table 2 shows the number of women initially showing erythema, edema or friability according to the initial cultures for $C$ trachomatis. Women from whom $C$ trachomatis was cultured were significantly more likely to show at least two of the three manifestations $(\mathrm{P}=0.05)$. All three women with $\mathrm{N}$ gonorrhoeae but not $C$ trachomatis had at least one of these three findings (two with one and one with three).

Cervical polymorphonuclear leukocytes: To be enrolled in the study women had to have 10 or more polymorphonuclear leukocytes per oil immersion field in cervical secretions. At the initial visit 50 or more polymorphonuclear leukocytes per oil immersion field were present in nine women with $C$ trachomatis and four women without $C$ trachomatis (two-tailed Fisher's exact test, $\mathrm{P}=0.08$ ).

Erythrocyte sedimentation rate, antibody to $C$ trachomatis and Pap smear results: There were no significant differences in erythrocyte sedimentation rates among women with and without $C$ trachomatis; rates were more than $10 \mathrm{~mm} / \mathrm{h}$ in five of 19 with detectable $C$ trachomatis (26\%) and in 10 of 23 without (43\%). One in each group had rates above $30 \mathrm{~mm} / \mathrm{h}$.

Among those with positive cultures for $C$ trachomatis, immunoglobulin $\mathrm{G}$ antibody to $C$ trachomatis at a titre of $1: 32$ or greater was present in 13 of $19(68 \%)$ compared with 10 of $23(43 \%)$ with negative cultures for $C$ trachomatis (two-tailed Fisher's exact test, $\mathrm{P}=0.19$ ). Five women with $C$ trachomatis (26\%) and nine women without C trachomatis (39\%) lacked detectable antibody.

Satisfactory Pap smears were available from 35 women (four women showed dysplastic cells). Three initially had positive cultures for $C$ trachomatis. At follow-up, dysplastic cells were not seen in two patients who initially had $C$ trachomatis isolated, and persisted in two (one with $C$ trachomatis initially).

Clinical response to treatment: Irrespective of treatment regimen or initial microbiology, mucopurulent discharge had almost always disappeared by the first follow-up and remained absent throughout the remainder of follow-up. It was noted in only one woman at the first follow-up, two others at the second, none at the third and another at the fourth. Thus, even when present at follow-up, it was only noted transiently. None of the women with mucopurulent discharge had positive cultures for C trachomatis or Ngonorrhoeae at follow-up.

Erythema, edema and friability tended to improve. although there was often little or no change by the first follow-up visit. In women with two or more follow-up visits, by the last visit none of these three symptoms was present in 19, one was present in six, two were present in six and all three were present in one (she had bacterial vaginosis but no mucopurulent discharge and normal numbers of polymorphonuclear leukocytes on Gram stain of cervical secretions). There was virtually no difference in findings between those with and without initial detection of $C$ trachomatis or according to treatment regimen.

The number of polymorphonuclear leukocytes in cervical secretions at follow-up was a poor guide to response. At 11 follow-up visits there were 50 or more 
polymorphonuclear leukocytes, but at only two of these visits were cultures positive for $C$ trachomatis. Of the 39 women with evaluable smears at the first follow-up, all but six still had at least 10 polymorphonuclear leukocytes per oil immersion field. As well, in second or subsequent follow-up visits with negative cultures for $C$ trachomatis, 10 or more polymorphonuclear leukocytes were found in 38 of 58 visits (66\%). There was no apparent trend with either initial microbiology or regimen used.

Microbiological response to treatment: For women who initially had a positive culture for $C$ trachomatis and follow-up to microbiological failure or for four or more weeks post treatment, cultures became and stayed negative in seven of eight on minocycline $100 \mathrm{mg}$ and five of six on minocycline $200 \mathrm{mg}$. Both women who ultimately had a positive culture post treatment had had an intervening negative culture and were re-exposed to untreated partners. Among the 12 considered to be cures, follow-up exceeded seven weeks post treatment in 10 .

All four women with $\mathrm{N}$ gonorrhoeae received minocycline $100 \mathrm{mg}$. One was lost to follow-up, but the other three had negative cultures post treatment with followup for 12, 22 and 105 days.

Adverse reactions: Adverse reactions were frequent and contributed to premature cessation of the study because they were significantly more frequent on the $200 \mathrm{mg}$ at bedtime regimen (Table 3). The most frequent adverse reactions were dizziness and mood alterations, but severe nausea was noted by one patient on $200 \mathrm{mg}$.

\section{DISCUSSION}

In this population of women attending a sexually transmitted disease clinic, use of the less rigorous definition of cervicitis recommended in Canadian guidelines was helpful in identifying a group of women at increased risk of infection with both $C$ trachomatis and $N$ gonorrhoeae. In this population, empiric treatment for women in whom a mucopurulent cervical discharge is detected and there are 10 or more polymorphonuclear leukocytes per oil immersion field of cervical secretions should include therapy directed against both pathogens. All three women infected with $\mathrm{Ngonor}$ rhoeae and negative cultures for $C$ trachomatis had at least one of the manifestations of erythema or edema in an area of ectopy, or induced mucosal bleeding, but the number of women with $\mathrm{N}$ gonorrhoeae was too small to test the hypothesis that significant numbers of women with gonococcal infection would be missed using the more rigid American definition. Women with $C$ trachomatis infection were significantly more likely to have at least two of the three findings.

The distribution of other recognized genital pathogens was similar between those with and without detection of $C$ trachomatis. Almost one-half of the women had neither $C$ trachomatis nor $N$ gonorrhoeae detected. However, there was no apparent difference in response to
TABLE 3

Reported adverse reactions on 100 and $200 \mathrm{mg}$ dose regimens of minocycline

\begin{tabular}{cccc}
\hline & $100 \mathbf{~ m g ~ ( n = 2 4 )}$ & $\mathbf{2 0 0} \mathbf{m g}(\mathrm{n}=20)$ & P Value* $^{*}$ \\
\hline Any & $6(25 \%)$ & $18(90 \%)$ & $<0.001$ \\
Severe & $1(4 \%)$ & $6(30 \%)$ & 0.05 \\
Dizziness & $3(13 \%)$ & $16(80 \%)$ & $<0.001$ \\
Severe & 0 & $5(25 \%)$ & 0.03 \\
Nausea & $4(17 \%)$ & $7(35 \%)$ & 0.29 \\
Altered mood & $2(8 \%)$ & $7(35 \%)$ & 0.07 \\
Severe & $1(4 \%)$ & $3(15 \%)$ & 0.47 \\
\hline
\end{tabular}

"Two-tailed Fisher exact test

treatment in these women, suggesting that minocycline therapy was acting against an antimicrobial susceptible etiology or that the detectable clinical manifestations are self-limited.

We cannot comment extensively on initial correlations between the number of polymorphonuclear leukocytes and the presence or absence of $C$ trachomatis because women had to have at least 10 polymorphonuclear leukocytes per oil immersion field to be enrolled. The possibility of a beta error is large, but although very high numbers correlated slightly with detection of $C$ trachomatis, the findings were not statistically significant. It is very evident in this study, however, that number of polymorphonuclear leukocytes in cervical secretions is a very poor guide to response to treatment and did not correlate with either microbiology or clinical manifestations at follow-up. As a sole criterion they should not be used as an indication for retreatment.

The number of women with $C$ trachomatis who were treated and followed is small. However, with the exception of two who were known to have been re-exposed to infected partners, all were cured, including those on the lower dose regimen, providing further support for the efficacy of lower doses of tetracycline than the usually recommended regimens of tetracycline $500 \mathrm{mg}$ four times daily or doxycycline $100 \mathrm{mg}$ twice daily. Proof in women that lower dose regimens are as effective as higher dose regimens will require a much larger study.

This study clearly shows that if lower dose regimens continue to be effective, there can be significant benefits to patients in terms of decreased adverse reactions. Adverse reactions were a major problem on the $200 \mathrm{mg}$ regimen. This was not an unexpected problem, although it was hoped that adminstration at bedtime would decrease the risk of reactions. It appears that doses of minocycline should not exceed $100 \mathrm{mg}$ daily in women. In a previous larger study in men, $100 \mathrm{mg}$ twice daily was tolerated less well than $100 \mathrm{mg}$ once daily, but the adverse reactions were not as disruptive as those encountered in the study patients (8). In a recently completed study, minocycline $100 \mathrm{mg}$ once daily was significantly better tolerated than doxycycline 100 mg twice daily in both men and women with comparable efficacy (12). 
ACKNOWLEDGEMENT: The support of Cyanamid Canada Inc is gratefully acknowledged.

\section{REFERENCES}

1. Holmes KK. Lower genital tract infections in women: Cystitis, urethritis, vulvovaginitis, and cervicitis. In: Holmes KK, Mardh PA, Sparling PF, et al, eds. Sexually Transmitted Diseases, 2nd edn. New York: McGraw-Hill Book Company, 1990:527-45.

2. Paavonen J, Critchlow CW, DeRouen T, et al. Etiology of cervical inflammation. Am J Obstet Gynecol 1986:154:556-64.

3. Brunham RC, Paavonen J, Stevens CE, et al. Mucopurulent cervicitis - the ignored counterpart in women of urethritis in men. $\mathrm{N}$ Engl $\mathrm{J}$ Med 1984:311:1-6.

4. Centers for Disease Control. 1989 Sexually Transmitted Diseases treatment guidelines. Morbid Mortal Wkly Rep 1989;38(S8):v-43.

5. MacDonald N, Bowie WR, Read S, eds. 1988 Canadian guidelines for the treatment of sexually transmitted diseases in neonates, children, adolescents, and adults. Can Dis Wkly Rep 1988;14S2:1-20.
6. Bowie WR, MacDonald NE, eds. 1989 Canadian guidelines for the diagnosis and management of sexually transmitted diseases, by syndrome, in children, adolescents and adults. Can Dis Wkly Rep 1989;15Sl:1-33.

7. Bowie WR. Epidemiology and therapy of Chlamydia trachomatis infections. Drugs 1984;27:459-68.

8. Bowie WR, Alexander ER, Stimson JB, Floyd JF, Holmes KK. Therapy for nongococcal urethritis: Double-blind randomized comparison of two doses and two durations of minocycline. Ann Intern Med 1981;95:306-11.

9. Bowie WR, Willetts V. Suboptiminal efficacy of erythromycin and tetracycline against vaginal Ureaplasma urealyticum. Sex Transm Dis 1987;14:88-91.

10. Wang SP, Grayston JT. Human serology in Chlamydia trachomatis infection with microimmunofluorescence. $J$ Infect Dis 1974;130:388-97.

11. Guijon FB, Paraskevas M, Brunham R. The association of sexually transmitted diseases with cervical intraepithelial neoplasia: A case-control study. Am J Obstet Gynecol $1985 ; 151: 185-90$

12. Romanowski B, Talbot H, Stadnyk M, Bowie WR. Minocycline vs doxycycline in the treatment of nongonococcal urethritis and mucopurulent cervicitis. Ann Intern Med. (In press) 


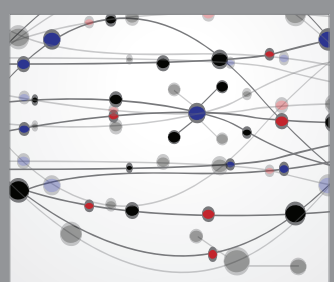

The Scientific World Journal
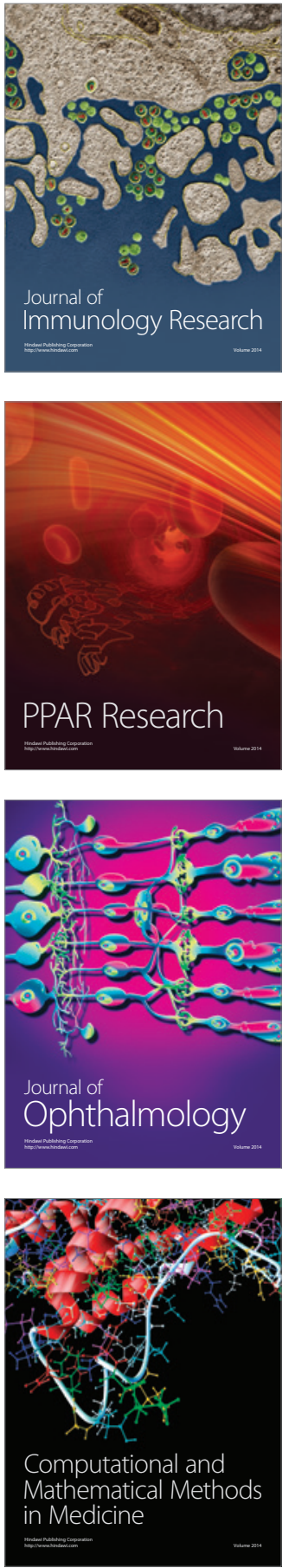

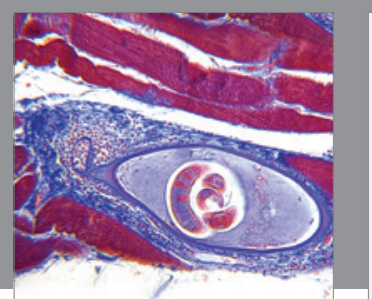

Gastroenterology Research and Practice

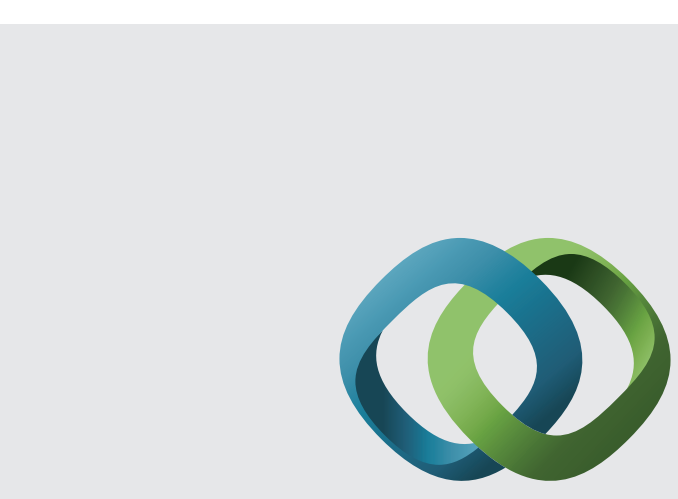

\section{Hindawi}

Submit your manuscripts at

http://www.hindawi.com
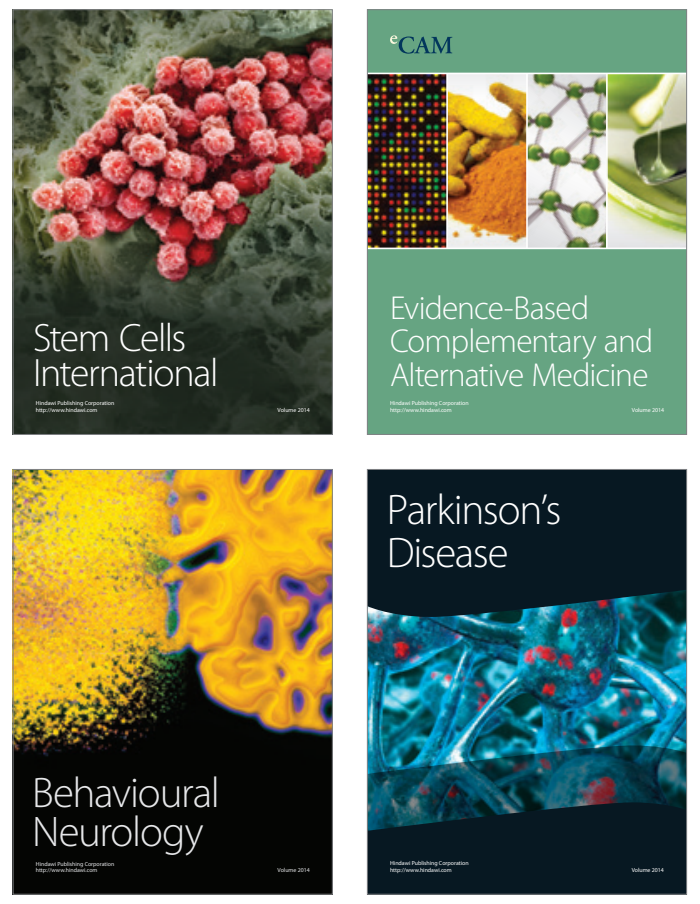
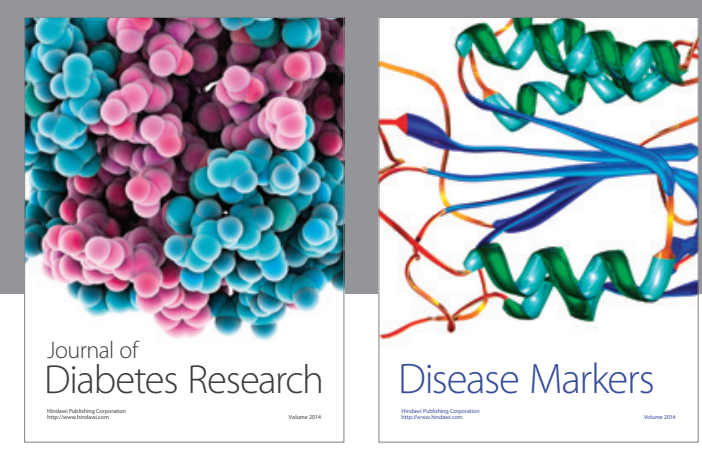

Disease Markers
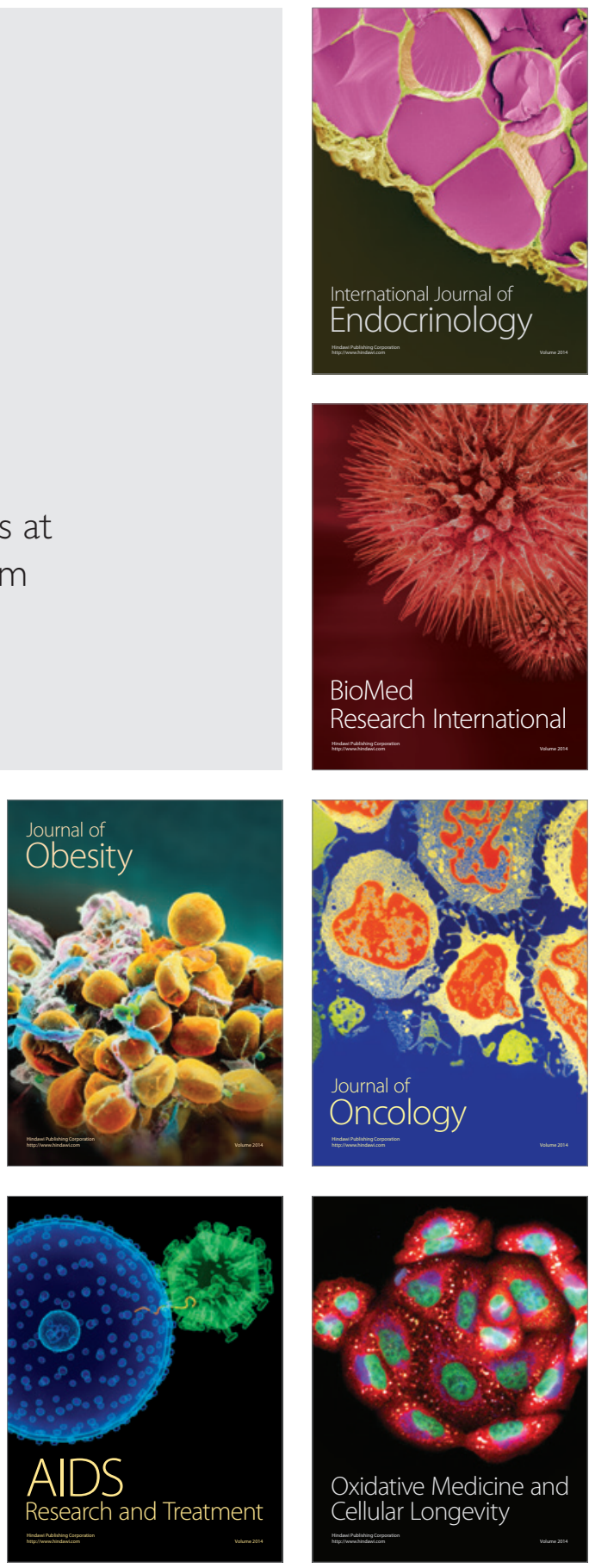